\title{
Nonexistence and existence results for a class of fourth-order difference mixed boundary value problems
}

\author{
Xiaoqing Deng
}

Received: 14 May 2013 / Published online: 2 August 2013

(C) The Author(s) 2013. This article is published with open access at Springerlink.com

\begin{abstract}
In this paper, a class of fourth-order nonlinear difference equations are considered. By making use of the critical point method, we establish various sets of sufficient conditions for the nonexistence and existence of solutions for mixed boundary value problems and give some new results. Our results successfully complement the existing results in the literature.
\end{abstract}

Keywords Nonexistence and existence - Mixed boundary value problems . Fourth-order $\cdot$ Mountain Pass lemma $\cdot$ Discrete variational theory

Mathematics Subject Classification (2000) 39A10

\section{Introduction}

Below $\mathbf{N}, \mathbf{Z}$ and $\mathbf{R}$ denote the sets of all natural numbers, integers and real numbers respectively. $k$ is a positive integer. For any $a, b \in \mathbf{Z}$, define $\mathbf{Z}(a)=$ $\{a, a+1, \ldots\}, \mathbf{Z}(a, b)=\{a, a+1, \ldots, b\}$ when $a<b . \Delta$ is the forward difference operator $\Delta u_{n}=u_{n+1}-u_{n}, \Delta^{2} u_{n}=\Delta\left(\Delta u_{n}\right)$. Besides, $*$ denotes the transpose of a vector.

Recently, difference equations have attracted the interest of many researchers since they provided a natural description of several discrete models. Such discrete models are often investigated in various fields of science and technology such as computer

This project is supported by Specialized Research Fund for the Natural Science Foundation of Hunan Province of China (Grant No. 10JJ5064) and Humanities and Social Sciences in general planning Fund of Ministry of Education of China (Grant No. 11YJAZH039).

X. Deng (凶)

School of Mathematics and Statistics, Hunan University of Commerce, Changsha 410205, China

e-mail: dengxq924@163.com 
science, economics, neural networks, ecology, cybernetics, biological systems, optimal control, and population dynamics. These studies cover many of the branches of difference equations, such as stability, attractivity, periodicity, oscillation, and boundary value problems, see [8, 17-19, 23, 26, 27, 29, 34, 42] and the references therein.

The present paper considers the fourth-order nonlinear difference equation

$$
\Delta^{2}\left(p_{n-1} \Delta^{2} u_{n-2}\right)-\Delta\left(q_{n}\left(\Delta u_{n-1}\right)^{\delta}\right)+r_{n} u_{n}^{\delta}=f\left(n, u_{n}\right), \quad n \in \mathbf{Z}(1, k),
$$

with boundary value conditions

$$
\Delta u_{-1}=\Delta u_{0}=0, \quad u_{k+1}=u_{k+2}=0,
$$

where $p_{n}$ is nonzero and real valued for each $n \in \mathbf{Z}(0, k+1),\left\{q_{n}\right\}_{n \in \mathbf{Z}(1, k+1)}$ and $\left\{r_{n}\right\}_{n \in \mathbf{Z}(1, k)}$ are real sequences, $\delta$ is the ratio of odd positive integers, $f \in C\left(\mathbf{R}^{2}, \mathbf{R}\right)$.

We may think of (1.1) with (1.2) as being a discrete analogue of the following fourth-order nonlinear differential equation

$$
\left[p(t) u^{\prime \prime}(t)\right]^{\prime \prime}-\left[q(t) u^{\prime}(t)\right]^{\prime}=f(t, u(t)), \quad t \in[a, b],
$$

with boundary value conditions

$$
u(a)=u^{\prime}(a)=0, \quad u(b)=u^{\prime}(b)=0 .
$$

Equation (1.3) includes the following equation

$$
u^{(4)}(t)=f(t, u(t)), \quad t \in \mathbf{R},
$$

which is used to describe the bending of an elastic beam; see, for example, [5, 16, $20,22,25,39]$ and the references therein. Owing to its importance in physics, many methods are applied to study fourth-order boundary value problems by many authors.

In recent years, the study of boundary value problems for differential equations develops at relatively rapid rate. By using various methods and techniques, such as fixed point theory, topological degree theory, coincidence degree theory, a series of existence results of nontrivial solutions for differential equations have been obtained in literatures, we refer to [2-4, 7, 21, 37]. And critical point theory is also an important tool to deal with problems on differential equations [11, 14, 15, 28, 33, 43]. Only since 2003, critical point theory has been employed to establish sufficient conditions on the existence of periodic solutions of difference equations. By using the critical point theory, Guo and Yu [17-19] and Shi et al. [35] have successfully proved the existence of periodic solutions of second-order nonlinear difference equations. We also refer to $[40,41]$ for the discrete boundary value problems. Compared to firstorder or second-order difference equations, the study of higher-order equations, and in particular, fourth-order equations, has received considerably less attention (see, for example, $[9,10,13,31,32,36,38]$ and the references contained therein). Yan, Liu [38] in 1997 and Thandapani, Arockiasamy [36] in 2001 studied the following fourth-order difference equation of form,

$$
\Delta^{2}\left(p_{n} \Delta^{2} u_{n}\right)+f\left(n, u_{n}\right)=0, \quad n \in \mathbf{Z} .
$$


The authors obtain criteria for the oscillation and nonoscillation of solutions for equation (1.6). In 2005, Cai, Yu and Guo [6] have obtained some criteria for the existence of periodic solutions of the fourth-order difference equation

$$
\Delta^{2}\left(p_{n-2} \Delta^{2} u_{n-2}\right)+f\left(n, u_{n}\right)=0, \quad n \in \mathbf{Z} .
$$

In 1995, Peterson and Ridenhour considered the disconjugacy of equation (1.7) when $p_{n} \equiv 1$ and $f\left(n, u_{n}\right)=q_{n} u_{n}$ (see [31]).

The boundary value problem (BVP) for determining the existence of solutions of difference equations has been a very active area of research in the last twenty years, and for surveys of recent results, we refer the reader to the monographs by Agarwal et al. [1, 12, 24, 29, 34]. We use the critical point theory to give some sufficient conditions for the nonexistence and existence of solutions for the BVP (1.1) with (1.2). We shall study the suplinear and sublinear cases. The main idea in this paper is to transfer the existence of the BVP (1.1) with (1.2) into the existence of the critical points of some functional. The proof is based on the notable Mountain Pass lemma in combination with variational technique. The purpose of this paper is two-folded. On one hand, we shall further demonstrate the powerfulness of critical point theory in the study of solutions for boundary value problems of difference equations. On the other hand, we shall complement existing ones. The motivation for the present work stems from the recent paper in [11].

Let

$$
\begin{array}{ll}
\bar{p}=\max \left\{p_{n}: n \in \mathbf{Z}(1, k+1)\right\}, & \underline{p}=\min \left\{p_{n}: n \in \mathbf{Z}(1, k+1)\right\}, \\
\bar{q}=\max \left\{q_{n}: n \in \mathbf{Z}(2, k+1)\right\}, & \underline{q}=\min \left\{q_{n}: n \in \mathbf{Z}(2, k+1)\right\}, \\
\bar{r}=\max \left\{r_{n}: n \in \mathbf{Z}(1, k)\right\}, & \underline{r}=\min \left\{r_{n}: n \in \mathbf{Z}(1, k)\right\} .
\end{array}
$$

Our main results are as follows.

Theorem 1.1 Assume that the following hypotheses are satisfied:

(p) for any $n \in Z(1, k+1), p_{n}<0$;

(q) for any $n \in \boldsymbol{Z}(2, k+1), q_{n} \leq 0$;

(r) for any $n \in \boldsymbol{Z}(1, k), r_{n} \leq 0$;

$\left(F_{1}\right)$ there exists a functional $F(n, v) \in C^{1}(\boldsymbol{Z} \times \boldsymbol{R}, \boldsymbol{R})$ with $F(0, \cdot)=0$ such that

$$
\frac{\partial F(n, v)}{\partial v}=f(n, v), \quad \forall n \in Z(1, k)
$$

$\left(F_{2}\right)$ there exists a constant $M_{0}>0$ for all $(n, v) \in \boldsymbol{Z}(1, k) \times \boldsymbol{R}$ such that $|f(n, v)| \leq$ $M_{0}$.

Then the BVP (1.1) with (1.2) possesses at least one solution.

Remark 1.1 Assumption $\left(F_{2}\right)$ implies that there exists a constant $M_{1}>0$ such that $\left(F_{2}^{\prime}\right)|F(n, v)| \leq M_{1}+M_{0}|v|, \forall(n, v) \in \mathbf{Z}(1, k) \times \mathbf{R}$. 
Theorem 1.2 Suppose that $\left(F_{1}\right)$ and the following hypotheses are satisfied:

$\left(p^{\prime}\right)$ for any $n \in \boldsymbol{Z}(1, k+1), p_{n}>0$;

$\left(q^{\prime}\right)$ for any $n \in \boldsymbol{Z}(2, k+1), q_{n} \geq 0$;

$\left(r^{\prime}\right)$ for any $n \in \mathbf{Z}(1, k), r_{n} \geq 0$;

$\left(F_{3}\right)$ there exists a functional $F(n, v) \in C^{1}(\boldsymbol{Z} \times \boldsymbol{R}, \boldsymbol{R})$ such that

$$
\lim _{v \rightarrow 0} \frac{F(n, v)}{|v|}=0, \quad \forall n \in \boldsymbol{Z}(1, k) ;
$$

$\left(F_{4}\right)$ there exists a constant $\beta>\max \{2, \delta+1\}$ such that for any $n \in \boldsymbol{Z}(1, k)$,

$$
0<v f(n, v)<\beta F(n, v), \quad \forall v \neq 0 .
$$

Then the BVP (1.1) with (1.2) possesses at least two nontrivial solutions.

Remark 1.2 Assumption $\left(F_{4}\right)$ implies that there exist constants $a_{1}>0$ and $a_{2}>0$ such that $\left(F_{4}^{\prime}\right) F(n, v)>a_{1}|v|^{\beta}-a_{2}, \forall n \in \mathbf{Z}(1, k)$.

Theorem 1.3 Suppose that $\left(p^{\prime}\right),\left(q^{\prime}\right),\left(r^{\prime}\right),\left(F_{1}\right)$ and the following assumption are satisfied:

( $\left.F_{5}\right)$ there exist constants $R>0$ and $1<\alpha<2$ such that for $n \in Z(1, k)$ and $|v| \geq R$,

$$
0<f(n, v) \leq \alpha F(n, v) .
$$

Then the BVP (1.1) with (1.2) possesses at least one solution.

Remark 1.3 Assumption ( $\left.F_{5}\right)$ implies that for each $n \in \mathbf{Z}(1, k)$ there exist constants $a_{3}>0$ and $a_{4}>0$ such that

$\left(F_{5}^{\prime}\right) \quad F(n, v) \leq a_{3}|v|^{\alpha}+a_{4}, \forall(n, v) \in \mathbf{Z}(1, k) \times \mathbf{R}$.

Theorem 1.4 Suppose that $(p),(q),(r),\left(F_{1}\right)$ and the following assumption are satisfied:

$\left(F_{6}\right) \quad v f(n, v)>0$, for $v \neq 0, \forall n \in \boldsymbol{Z}(1, k)$.

Then the BVP (1.1) with (1.2) has no nontrivial solutions.

Remark 1.4 In the existing literature, results on the nonexistence of solutions of discrete boundary value problems are very scarce. Hence, Theorem 1.4 complements existing ones.

The remainder of this paper is organized as follows. First, in Sect. 2, we shall establish the variational framework for the BVP (1.1) with (1.2) and transfer the problem of the existence of the BVP (1.1) with (1.2) into that of the existence of critical points of the corresponding functional. Some related fundamental results will also be recalled. Then, in Sect. 3, we shall complete the proof of the results by using the 
critical point method. Finally, in Sect. 4, we shall give three examples to illustrate the main results.

About the basic knowledge for variational methods, we refer the reader to [28, 30 , $33,43]$.

\section{Variational structure and some lemmas}

In order to apply the critical point theory, we shall establish the corresponding variational framework for the BVP (1.1) with (1.2) and give some lemmas which will be of fundamental importance in proving our main results. First, we state some basic notations.

Let $\mathbf{R}^{k}$ be the real Euclidean space with dimension $k$. Define the inner product on $\mathbf{R}^{k}$ as follows:

$$
\langle u, v\rangle=\sum_{j=1}^{k} u_{j} v_{j}, \quad \forall u, v \in \mathbf{R}^{k},
$$

by which the norm $\|\cdot\|$ can be induced by

$$
\|u\|=\left(\sum_{j=1}^{k} u_{j}^{2}\right)^{\frac{1}{2}}, \quad \forall u \in \mathbf{R}^{k} .
$$

On the other hand, we define the norm $\|\cdot\|_{r}$ on $\mathbf{R}^{k}$ as follows:

$$
\|u\|_{r}=\left(\sum_{j=1}^{k}\left|u_{j}\right|^{r}\right)^{\frac{1}{r}},
$$

for all $u \in \mathbf{R}^{k}$ and $r>1$.

Since $\|u\|_{r}$ and $\|u\|_{2}$ are equivalent, there exist constants $c_{1}, c_{2}$ such that $c_{2} \geq$ $c_{1}>0$, and

$$
c_{1}\|u\|_{2} \leq\|u\|_{r} \leq c_{2}\|u\|_{2}, \quad \forall u \in \mathbf{R}^{k} .
$$

Clearly, $\|u\|=\|u\|_{2}$. For any $u=\left(u_{1}, u_{2}, \ldots, u_{k}\right)^{*} \in \mathbf{R}^{k}$, for the BVP (1.1) with (1.2), consider the functional $J$ defined on $\mathbf{R}^{k}$ as follows:

$$
\begin{aligned}
J(u)= & \frac{1}{2} \sum_{n=1}^{k} p_{n+1}\left(\Delta^{2} u_{n}\right)^{2}+\frac{1}{\delta+1} \sum_{n=1}^{k} q_{n+1}\left(\Delta u_{n}\right)^{\delta+1}+\frac{1}{\delta+1} \sum_{n=1}^{k} r_{n} u_{n}^{\delta+1} \\
& -\sum_{n=1}^{k} F\left(n, u_{n}\right)+\frac{1}{2} p_{1}\left(\Delta u_{1}\right)^{2}
\end{aligned}
$$

where

$$
\frac{\partial F(n, v)}{\partial v}=f(n, v), \quad \Delta u_{-1}=\Delta u_{0}=0, \quad u_{k+1}=u_{k+2}=0 .
$$


Clearly, $J \in C^{1}\left(\mathbf{R}^{k}, \mathbf{R}\right)$ and for any $u=\left\{u_{n}\right\}_{n=1}^{k}=\left(u_{1}, u_{2}, \ldots, u_{k}\right)^{*}$, by using $\Delta u_{-1}=\Delta u_{0}=0, u_{k+1}=u_{k+2}=0$, we can compute the partial derivative as

$$
\frac{\partial J}{\partial u_{n}}=\Delta^{2}\left(p_{n-1} \Delta^{2} u_{n-2}\right)-\Delta\left(q_{n}\left(\Delta u_{n-1}\right)^{\delta}\right)+r_{n} u_{n}^{\delta}-f\left(n, u_{n}\right), \quad \forall n \in \mathbf{Z}(1, k) .
$$

Thus, $u$ is a critical point of $J$ on $\mathbf{R}^{k}$ if and only if

$$
\Delta^{2}\left(p_{n-1} \Delta^{2} u_{n-2}\right)-\Delta\left(q_{n}\left(\Delta u_{n-1}\right)^{\delta}\right)+r_{n} u_{n}^{\delta}=f\left(n, u_{n}\right), \quad \forall n \in \mathbf{Z}(1, k) .
$$

We reduce the existence of the BVP (1.1) with (1.2) to the existence of critical points of $J$ on $\mathbf{R}^{k}$. That is, the functional $J$ is just the variational framework of the BVP (1.1) with (1.2).

Let $P$ and $Q$ be the $k \times k$ matrices defined by

$$
\begin{aligned}
P & =\left(\begin{array}{ccccccccc}
6 & -4 & 1 & 0 & 0 & \cdots & 0 & 0 & 0 \\
-4 & 6 & -4 & 1 & 0 & \cdots & 0 & 0 & 0 \\
1 & -4 & 6 & -4 & 1 & \cdots & 0 & 0 & 0 \\
0 & 1 & -4 & 6 & -4 & \cdots & 0 & 0 & 0 \\
\vdots & \vdots & \vdots & \vdots & \vdots & \ddots & \vdots & \vdots & \vdots \\
0 & 0 & 0 & 0 & 0 & \cdots & 6 & -4 & 1 \\
0 & 0 & 0 & 0 & 0 & \cdots & -4 & 6 & -4 \\
0 & 0 & 0 & 0 & 0 & \cdots & 1 & -4 & 6
\end{array}\right), \\
Q & =\left(\begin{array}{cccccccc}
2 & -1 & 0 & \cdots & 0 & 0 \\
-1 & 2 & -1 & \cdots & 0 & 0 \\
0 & -1 & 2 & \cdots & 0 & 0 \\
\vdots & \vdots & \vdots & \ddots & \vdots & \vdots \\
0 & 0 & 0 & \cdots & 2 & -1 \\
0 & 0 & 0 & \cdots & -1 & 2
\end{array}\right) .
\end{aligned}
$$

Clearly, $P$ and $Q$ are positive definite. Let $\lambda_{1}, \lambda_{2}, \ldots, \lambda_{k}$ be the eigenvalues of $P, \tilde{\lambda}_{1}, \tilde{\lambda}_{2}, \ldots, \tilde{\lambda}_{k}$ be the eigenvalues of $Q$. Applying matrix theory, we know $\lambda_{j}>$ $0, \tilde{\lambda}_{j}>0, j=1,2, \ldots, k$. Without loss of generality, we may assume that

$$
\begin{aligned}
& 0<\lambda_{1} \leq \lambda_{2} \leq \cdots \leq \lambda_{k} \\
& 0<\tilde{\lambda}_{1} \leq \tilde{\lambda}_{2} \leq \cdots \leq \tilde{\lambda}_{k} .
\end{aligned}
$$

Let $E$ be a real Banach space, $J \in C^{1}(E, \mathbf{R})$, i.e., $J$ is a continuously Fréchetdifferentiable functional defined on $E$. $J$ is said to satisfy the Palais-Smale condition (P.S. condition for short) if any sequence $\left\{u^{(l)}\right\} \subset E$ for which $\left\{J\left(u^{(l)}\right)\right\}$ is bounded and $J^{\prime}\left(u^{(l)}\right) \rightarrow 0(l \rightarrow \infty)$ possesses a convergent subsequence in $E$.

Let $B_{\rho}$ denote the open ball in $E$ about 0 of radius $\rho$ and let $\partial B_{\rho}$ denote its boundary.

Lemma 2.1 (Mountain Pass lemma [33]) Let $E$ be a real Banach space and $J \in$ $C^{1}(E, \mathbf{R})$ satisfy the P.S. condition. If $J(0)=0$ and 
$\left(J_{1}\right)$ there exist constants $\rho, a>0$ such that $\left.J\right|_{\partial B_{\rho}} \geq a$, and

$\left(J_{2}\right)$ there exists $e \in E \backslash B_{\rho}$ such that $J(e) \leq 0$.

Then $J$ possesses a critical value $c \geq a$ given by

$$
c=\inf _{g \in \Gamma} \max _{s \in[0,1]} J(g(s)),
$$

where

$$
\Gamma=\{g \in C([0,1], E) \mid g(0)=0, g(1)=e\} .
$$

Lemma 2.2 Suppose that $\left(p^{\prime}\right),\left(q^{\prime}\right),\left(r^{\prime}\right),\left(F_{1}\right),\left(F_{3}\right)$ and $\left(F_{4}\right)$ are satisfied. Then the functional $J$ satisfies the P.S. condition.

Proof Let $u^{(l)} \in \mathbf{R}^{k}, l \in \mathbf{Z}(1)$ be such that $\left\{J\left(u^{(l)}\right)\right\}$ is bounded. Then there exists a positive constant $M_{2}$ such that

$$
-M_{2} \leq J\left(u^{(l)}\right) \leq M_{2}, \quad \forall l \in \mathbf{N}
$$

By $\left(F_{4}^{\prime}\right)$, we have

$$
\begin{aligned}
-M_{2} \leq & J\left(u^{(l)}\right) \\
= & \frac{1}{2} \sum_{n=1}^{k} p_{n+1}\left(\Delta^{2} u_{n}^{(l)}\right)^{2}+\frac{1}{\delta+1} \sum_{n=1}^{k} q_{n+1}\left(\Delta u_{n}^{(l)}\right)^{\delta+1} \\
& +\frac{1}{\delta+1} \sum_{n=1}^{k} r_{n}\left(u_{n}^{(l)}\right)^{\delta+1}-\sum_{n=1}^{k} F\left(n, u_{n}^{(l)}\right)+\frac{1}{2} p_{1}\left(\Delta u_{1}^{(l)}\right)^{2} \\
\leq & \frac{\bar{p}}{2} \sum_{n=1}^{k}\left(u_{n+2}^{(l)}-2 u_{n+1}^{(l)}+u_{n}^{(l)}\right)^{2}+\frac{\bar{q} c_{2}^{\delta+1}}{\delta+1}\left\{\left[\sum_{n=1}^{k}\left(u_{n+1}^{(l)}-u_{n}^{(l)}\right)^{2}\right]^{\frac{1}{2}}\right\}^{\delta+1} \\
& +\frac{\bar{r} c_{2}^{\delta+1}}{\delta+1}\left\|u^{(l)}\right\|^{\delta+1}-a_{1} \sum_{n=1}^{k}\left|u_{n}^{(l)}\right|^{\beta}+a_{2} k+\bar{p}\left\|u^{(l)}\right\|^{2} \\
\leq & \frac{\bar{p}}{2}\left(u^{(l)}\right)^{*} P u^{(l)}+\frac{\bar{q} c_{2}^{\delta+1}}{\delta+1}\left[\left(u^{(l)}\right)^{*} Q u^{(l)}\right]^{\frac{\delta+1}{2}}+\frac{\bar{r} c_{2}^{\delta+1}}{\delta+1}\left\|u^{(l)}\right\|^{\delta+1} \\
& -a_{1} c_{1}^{\beta}\left\|u^{(l)}\right\|^{\beta}+a_{2} k+\bar{p}\left\|u^{(l)}\right\|^{2} \\
\leq & \left(\frac{\lambda_{k}}{2}+1\right) \bar{p}\left\|u^{(l)}\right\|^{2}+\frac{\bar{q} c_{2}^{\delta+1} \tilde{\lambda}_{k}^{\frac{\delta+1}{2}}}{\delta+1}\left\|u^{(l)}\right\|^{\delta+1}+\frac{\bar{r} c_{2}^{\delta+1}}{\delta+1}\left\|u^{(l)}\right\|^{\delta+1} \\
& -a_{1} c_{1}^{\beta}\left\|u^{(l)}\right\|^{\beta}+a_{2} k,
\end{aligned}
$$


where $u^{(l)}=\left(u_{1}^{(l)}, u_{2}^{(l)}, \ldots, u_{k}^{(l)}\right)^{*}, u^{(l)} \in \mathbf{R}^{k}$. That is,

$$
\begin{aligned}
& a_{1} c_{1}^{\beta}\left\|u^{(l)}\right\|^{\beta}-\left(\frac{\lambda_{k}}{2}+1\right) \bar{p}\left\|u^{(l)}\right\|^{2}-\frac{\bar{q} c_{2}^{\delta+1} \tilde{\lambda}_{k}^{\frac{\delta+1}{2}}}{\delta+1}\left\|u^{(l)}\right\|^{\delta+1}-\frac{\bar{r} c_{2}^{\delta+1}}{\delta+1}\left\|u^{(l)}\right\|^{\delta+1} \\
& \quad \leq M_{2}+a_{2} k .
\end{aligned}
$$

Since $\beta>\max \{2, \delta+1\}$, there exists a constant $M_{3}>0$ such that

$$
\left\|u^{(l)}\right\| \leq M_{3}, \quad \forall l \in \mathbf{N} .
$$

Therefore, $\left\{u^{(l)}\right\}$ is bounded on $\mathbf{R}^{k}$. As a consequence, $\left\{u^{(l)}\right\}$ possesses a convergence subsequence in $\mathbf{R}^{k}$. Thus the P.S. condition is verified.

\section{Proof of the main results}

In this section, we shall prove our main results by using the critical point theory.

Proof of Theorem 1.1 By $\left(F_{2}^{\prime}\right)$, for any $u=\left(u_{1}, u_{2}, \ldots, u_{k}\right)^{*} \in \mathbf{R}^{k}$, we have

$$
\begin{aligned}
J(u)= & \frac{1}{2} \sum_{n=1}^{k} p_{n+1}\left(\Delta^{2} u_{n}\right)^{2}+\frac{1}{\delta+1} \sum_{n=1}^{k} q_{n+1}\left(\Delta u_{n}\right)^{\delta+1}+\frac{1}{\delta+1} \sum_{n=1}^{k} r_{n} u_{n}^{\delta+1} \\
& -\sum_{n=1}^{k} F\left(n, u_{n}\right)+\frac{1}{2} p_{1}\left(\Delta u_{1}\right)^{2} \\
\leq & \frac{\bar{p}}{2} \sum_{n=1}^{k}\left(u_{n+2}-2 u_{n+1}+u_{n}\right)^{2}+M_{0} \sum_{n=1}^{k}\left|u_{n}\right|+M_{1} k \\
\leq & \frac{\bar{p}}{2} u^{*} P u+M_{0} \sqrt{k}\|u\|+M_{1} k \\
\leq & \frac{\bar{p} \lambda_{1}}{2}\|u\|^{2}+M_{0} \sqrt{k}\|u\|+M_{1} k \rightarrow-\infty
\end{aligned}
$$

as $\|u\| \rightarrow+\infty$.

The above inequality means that $-J(u)$ is coercive. By the continuity of $J(u)$, $J$ attains its maximum at some point, and we denote it $\breve{u}$, that is,

$$
J(\check{u})=\max \left\{J(u) \mid u \in \mathbf{R}^{k}\right\} .
$$

Clearly, $\check{u}$ is a critical point of the functional $J$. This completes the proof of Theorem 1.1.

Proof of Theorem 1.2 By $\left(F_{3}\right)$, for any $\epsilon=\frac{p \lambda_{1}}{4}\left(\lambda_{1}\right.$ can be referred to (2.6)), there exists $\rho>0$, such that

$$
|F(n, v)| \leq \frac{p \lambda_{1}}{4}|v|, \quad \forall n \in \mathbf{Z}(1, k),
$$

for $|v| \leq \rho$. 
For any $u=\left(u_{1}, u_{2}, \ldots, u_{k}\right)^{*} \in \mathbf{R}^{k}$ and $\|u\| \leq \rho$, we have $\left|u_{n}\right| \leq \rho, n \in \mathbf{Z}(1, k)$.

For any $n \in \mathbf{Z}(1, k)$,

$$
\begin{aligned}
J(u)= & \frac{1}{2} \sum_{n=1}^{k} p_{n+1}\left(\Delta^{2} u_{n}\right)^{2}+\frac{1}{\delta+1} \sum_{n=1}^{k} q_{n+1}\left(\Delta u_{n}\right)^{\delta+1}+\frac{1}{\delta+1} \sum_{n=1}^{k} r_{n} u_{n}^{\delta+1} \\
& -\sum_{n=1}^{k} F\left(n, u_{n}\right)+\frac{1}{2} p_{1}\left(\Delta u_{1}\right)^{2} \\
\geq & \frac{p}{2} \sum_{n=1}^{k}\left(u_{n+2}-2 u_{n+1}+u_{n}\right)^{2}-\frac{p \lambda_{1}}{4} \sum_{n=1}^{k}\left|u_{n}\right| \\
\geq & \frac{p}{2} u^{*} P u-\frac{p \lambda_{1}}{4}\|u\|^{2} \\
\geq & \frac{p \lambda_{1}}{2}\|u\|^{2}-\frac{p \lambda_{1}}{4}\|u\|^{2} \\
= & \frac{p \lambda_{1}}{4}\|u\|^{2},
\end{aligned}
$$

where $u=\left(u_{1}, u_{2}, \ldots, u_{k}\right)^{*}, u \in \mathbf{R}^{k}$.

Take $a=\frac{p \lambda_{1}}{4} \rho^{2}>0$. Therefore,

$$
J(u) \geq a>0, \quad \forall u \in \partial B_{\rho} .
$$

At the same time, we have also proved that there exist constants $a>0$ and $\rho>0$ such that $\left.J\right|_{\partial B_{\rho}} \geq a$. That is to say, $J$ satisfies the condition $\left(J_{1}\right)$ of the Mountain Pass lemma.

For our setting, clearly $J(0)=0$. In order to exploit the Mountain Pass lemma in critical point theory, we need to verify other conditions of the Mountain Pass lemma. By Lemma 2.2, $J$ satisfies the P.S. condition. So it suffices to verify the condition $\left(J_{2}\right)$.

From the proof of the P.S. condition, we know

$$
J(u) \leq\left(\frac{\lambda_{k}}{2}+1\right) \bar{p}\|u\|^{2}+\frac{\bar{q} c_{2}^{\delta+1} \tilde{\lambda}_{k}^{\frac{\delta+1}{2}}}{\delta+1}\|u\|^{\delta+1}+\frac{\bar{r} c_{2}^{\delta+1}}{\delta+1}\|u\|^{\delta+1}-a_{1} c_{1}^{\beta}\|u\|^{\beta}+a_{2} k .
$$

Since $\beta>\max \{2, \delta+1\}$, we can choose $\bar{u}$ large enough to ensure that $J(\bar{u})<0$.

By the Mountain Pass lemma, $J$ possesses a critical value $c \geq a>0$, where

$$
c=\inf _{h \in \Gamma} \sup _{s \in[0,1]} J(h(s))
$$

and

$$
\Gamma=\left\{h \in C\left([0,1], \mathbf{R}^{k}\right) \mid h(0)=0, h(1)=\bar{u}\right\} .
$$


Let $\tilde{u} \in \mathbf{R}^{k}$ be a critical point associated to the critical value $c$ of $J$, i.e., $J(\tilde{u})=c$. Similar to the proof of the P.S. condition, we know that there exists $\hat{u} \in \mathbf{R}^{k}$ such that

$$
J(\hat{u})=c_{\max }=\max _{s \in[0,1]} J(h(s)) .
$$

Clearly, $\hat{u} \neq 0$. If $\tilde{u} \neq \hat{u}$, then the conclusion of Theorem 1.2 holds. Otherwise, $\tilde{u}=\hat{u}$. Then $c=J(\tilde{u})=c_{\max }=\max _{s \in[0,1]} J(h(s))$. That is,

$$
\sup _{u \in \mathbf{R}^{k}} J(u)=\inf _{h \in \Gamma} \sup _{s \in[0,1]} J(h(s)) .
$$

Therefore,

$$
c_{\max }=\max _{s \in[0,1]} J(h(s)), \quad \forall h \in \Gamma .
$$

By the continuity of $J(h(s))$ with respect to $s, J(0)=0$ and $J(\bar{u})<0$ imply that there exists $s_{0} \in(0,1)$ such that

$$
J\left(h\left(s_{0}\right)\right)=c_{\max } .
$$

Choose $h_{1}, h_{2} \in \Gamma$ such that $\left\{h_{1}(s) \mid s \in(0,1)\right\} \cap\left\{h_{1}(s) \mid s \in(0,1)\right\}$ is empty, then there exists $s_{1}, s_{2} \in(0,1)$ such that

$$
J\left(h_{1}\left(s_{1}\right)\right)=J\left(h_{2}\left(s_{2}\right)\right)=c_{\max } .
$$

Thus, we get two different critical points of $J$ on $\mathbf{R}^{k}$ denoted by

$$
u^{1}=h_{1}\left(s_{1}\right), u^{2}=h_{2}\left(s_{2}\right) .
$$

The above argument implies that the BVP (1.1) with (1.2) possesses at least two nontrivial solutions. The proof of Theorem 1.2 is finished.

Proof of Theorem 1.3 We only need to find at least one critical point of the functional $J$ defined as in (2.5).

By $\left(F_{5}^{\prime}\right)$, for any $u=\left(u_{1}, u_{2}, \ldots, u_{k}\right)^{*} \in \mathbf{R}^{k}$, we have

$$
\begin{aligned}
J(u)= & \frac{1}{2} \sum_{n=1}^{k} p_{n+1}\left(\Delta^{2} u_{n}\right)^{2}+\frac{1}{\delta+1} \sum_{n=1}^{k} q_{n+1}\left(\Delta u_{n}\right)^{\delta+1}+\frac{1}{\delta+1} \sum_{n=1}^{k} r_{n} u_{n}^{\delta+1} \\
& -\sum_{n=1}^{k} F\left(n, u_{n}\right)+\frac{1}{2} p_{1}\left(\Delta u_{1}\right)^{2} \\
\geq & \frac{p}{2} \sum_{n=1}^{k}\left(u_{n+2}-2 u_{n+1}+u_{n}\right)^{2}-a_{3} \sum_{n=1}^{k}\left|u_{n}\right|^{\alpha}-a_{4} k \\
= & \frac{p}{2} u^{*} P u-a_{3}\left[\left(\sum_{n=1}^{k}\left|u_{n}\right|^{\alpha}\right)^{\frac{1}{\alpha}}\right]^{\alpha}-a_{4} k
\end{aligned}
$$




$$
\begin{aligned}
& \geq \frac{p \lambda_{1}}{2}\|u\|^{2}-a_{3} c_{2}^{\alpha}\left[\left(\sum_{n=1}^{k} u_{n}^{2}\right)^{\frac{1}{2}}\right]^{\alpha}-a_{4} k \\
& \geq \frac{p \lambda_{1}}{2}\|u\|^{2}-a_{3} c_{2}^{\alpha}\|u\|^{\alpha}-a_{4} k \rightarrow+\infty
\end{aligned}
$$

as $\|u\| \rightarrow+\infty$.

By the continuity of $J$, we know from the above inequality that there exist lower bounds of values of the functional. And this means that $J$ attains its minimal value at some point which is just the critical point of $J$ with the finite norm.

Proof of Theorem 1.4 Assume, for the sake of contradiction, that the BVP (1.1) with (1.2) has a nontrivial solution. Then $J$ has a nonzero critical point $u^{\star}$. Since

$$
\frac{\partial J}{\partial u_{n}}=\Delta^{2}\left(p_{n-1} \Delta^{2} u_{n-2}\right)-\Delta\left(q_{n}\left(\Delta u_{n-1}\right)^{\delta}\right)+r_{n} u_{n}^{\delta}-f\left(n, u_{n}\right),
$$

we get

$$
\begin{aligned}
& \sum_{n=1}^{k} f\left(n, u_{n}^{\star}\right) u_{n}^{\star} \\
& \quad=\sum_{n=1}^{k}\left[\Delta^{2}\left(p_{n-1} \Delta^{2} u_{n-2}^{\star}\right)-\Delta\left(q_{n}\left(\Delta u_{n-1}^{\star}\right)^{\delta}\right)+r_{n}\left(u_{n}^{\star}\right)^{\delta}\right] u_{n}^{\star} \\
& \quad=\sum_{n=1}^{k} p_{n+1}\left(\Delta^{2} u_{n}^{\star}\right)^{2}+\sum_{n=1}^{k} q_{n+1}\left(\Delta u_{n}^{\star}\right)^{\delta+1}+\sum_{n=1}^{k} r_{n}\left(u_{n}^{\star}\right)^{\delta+1}+p_{1}\left(\Delta u_{1}^{\star}\right)^{2} \leq 0 .
\end{aligned}
$$

On the other hand, it follows from $\left(F_{6}\right)$ that

$$
\sum_{n=1}^{k} f\left(n, u_{n}^{\star}\right) u_{n}^{\star}>0
$$

This contradicts (3.1) and hence the proof is complete.

\section{Examples}

As an application of Theorems 1.2, 1.3 and 1.4, we give four examples to illustrate our main results.

Example 4.1 For $n \in \mathbf{Z}(1, k)$, assume that

$$
\Delta^{4} u_{n-2}-\Delta\left(9^{n}\left(\Delta u_{n-1}\right)^{\delta}\right)+3^{n} u_{n}^{\delta}=\beta \varphi(n) u_{n}\left|u_{n}\right|^{\beta-2},
$$


with boundary value conditions (1.2), $\delta$ is the ratio of odd positive integers, $\beta>\max \{2, \delta+1\}, \varphi$ is continuously differentiable and $\varphi(n)>0, n \in \mathbf{Z}(1, k)$ with $\varphi(0)=0$. We have

$$
p_{n} \equiv 1, \quad q_{n}=9^{n}, \quad r_{n}=3^{n}, \quad f(n, v)=\beta \varphi(n) v|v|^{\beta-2}
$$

and

$$
F(n, v)=\varphi(n)|v|^{\beta} .
$$

It is easy to verify all the assumptions of Theorem 1.2 are satisfied and then the BVP (4.1) with (1.2) possesses at least two nontrivial solutions.

Example 4.2 For $n \in \mathbf{Z}(1, k)$, assume that

$$
\Delta^{2}\left(8^{n-1} \Delta^{2} u_{n-2}\right)-\Delta\left(6^{n}\left(\Delta u_{n-1}\right)^{\delta}\right)+5^{n} u_{n}^{\delta}=\alpha \psi(n) u_{n}\left|u_{n}\right|^{\alpha-2},
$$

with boundary value conditions (1.2), $\delta$ is the ratio of odd positive integers, $1<\alpha<$ $2, \psi$ is continuously differentiable and $\psi(n)>0, n \in \mathbf{Z}(1, k)$ with $\psi(0)=0$. We have

$$
p_{n}=8^{n}, \quad q_{n}=6^{n}, \quad r_{n}=5^{n}, \quad f(n, v)=\alpha \psi(n) v|v|^{\alpha-2}
$$

and

$$
F(n, v)=\psi(n)|v|^{\alpha} .
$$

It is easy to verify all the assumptions of Theorem 1.3 are satisfied and then the BVP (4.2) with (1.2) possesses at least one solution.

Example 4.3 For $n \in \mathbf{Z}(1, k)$, assume that

$$
-\Delta^{4} u_{n-2}+\Delta\left(9^{n}\left(\Delta u_{n-1}\right)^{\delta}\right)-8^{n} u_{n}^{\delta}=\frac{4}{3} u_{n}^{\frac{1}{3}},
$$

with boundary value conditions (1.2), $\delta$ is the ratio of odd positive integers. We have

$$
p_{n} \equiv-1, \quad q_{n}=-9^{n}, \quad r_{n}=-8^{n}, \quad f(n, v)=\frac{4}{3} v^{\frac{1}{3}}
$$

and

$$
F(n, v)=v^{\frac{4}{3}}
$$

It is easy to verify all the assumptions of Theorem 1.4 are satisfied and then the BVP (4.3) with (1.2) has no nontrivial solutions.

Example 4.4 For $n \in \mathbf{Z}(1, k)$, assume that

$$
\Delta^{4} u_{n-2}=4 u_{n}\left|u_{n}\right|^{3},
$$


with boundary value conditions (1.2). We have

$$
\begin{array}{ll}
\delta=1, \quad \beta=5, & p_{n} \equiv 1, \quad q_{n}=r_{n} \equiv 0, \\
f(n, v)=4 v|v|^{3}, & F(n, v)=\frac{4}{5}|v|^{5} .
\end{array}
$$

It is easy to verify all the assumptions of Theorem 1.2 are satisfied and then the BVP (4.4) with (1.2) possesses two nontrivial solutions $u_{n}=\sin \left(\frac{\pi}{2} n\right)$ and $u_{n}=\cos \left(\frac{\pi}{2} n\right)$.

Open Access This article is distributed under the terms of the Creative Commons Attribution License which permits any use, distribution, and reproduction in any medium, provided the original author(s) and the source are credited.

\section{References}

1. Agarwal, R.P.: Difference Equations and Inequalities: Theory, Methods and Applications. Marcel Dekker, New York (2000)

2. Ahlbrandt, C.D.: Dominant and recessive solutions of symmetric three term recurrences. J. Differ. Equ. 107(2), 238-258 (1994)

3. Anuradha, V., Maya, C., Shivaji, R.: Positive solutions for a class of nonlinear boundary value problems with Neumann-Robin boundary conditions. J. Math. Anal. Appl. 236(1), 94-124 (1999)

4. Arcoya, D.: Positive solutions for semilinear Dirichlet problems in an annulus. J. Differ. Equ. 94(2), 217-227 (1991)

5. Bai, Z., Wang, H.: On positive solutions of some nonlinear fourth-order beam equations. J. Math. Anal. Appl. 270(2), 357-368 (2002)

6. Cai, X.C., Yu, J.S., Guo, Z.M.: Existence of periodic solutions for fourth-order difference equations. Comput. Math. Appl. 50(1-2), 49-55 (2005)

7. Cecchi, M., Marini, M., Villari, G.: On the monotonicity property for a certain class of second order differential equations. J. Differ. Equ. 82(1), 15-27 (1989)

8. Chen, S.Z.: Disconjugacy, disfocality, and oscillation of second order difference equations. J. Differ. Equ. 107(2), 383-394 (1994)

9. Chen, P., Fang, H.: Existence of periodic and subharmonic solutions for second-order $p$-Laplacian difference equations. Adv. Differ. Equ. 2007, 1-9 (2007)

10. Chen, P., Tang, X.H.: Existence of infinitely many homoclinic orbits for fourth-order difference systems containing both advance and retardation. Appl. Math. Comput. 217(9), 4408-4415 (2011)

11. Chen, P., Tang, X.H.: New existence and multiplicity of solutions for some Dirichlet problems with impulsive effects. Math. Comput. Model. 55(3-4), 723-739 (2012)

12. Cull, P., Flahive, M., Robson, R.: Difference Equations: From Rabbits to Chaos. Springer, New York (2005)

13. Fang, H., Zhao, D.P.: Existence of nontrivial homoclinic orbits for fourth-order difference equations. Appl. Math. Comput. 214(1), 163-170 (2009)

14. Guo, C.J., O’Regan, D., Xu, Y.T., Agarwal, R.P.: Existence of subharmonic solutions and homoclinic orbits for a class of even higher order differential equations. Appl. Anal. 90(7), 1169-1183 (2011)

15. Guo, C.J., O’Regan, D., Xu, Y.T., Agarwal, R.P.: Existence and multiplicity of homoclinic orbits of a second-order differential difference equation via variational methods. Appl. Math. Inf. Mech. 4(1), $1-15$ (2012)

16. Guo, C.J., Xu, Y.T.: Existence of periodic solutions for a class of second order differential equation with deviating argument. J. Appl. Math. Comput. 28(1-2), 425-433 (2008)

17. Guo, Z.M., Yu, J.S.: Applications of critical point theory to difference equations. Fields Inst. Commun. 42, 187-200 (2004)

18. Guo, Z.M., Yu, J.S.: Existence of periodic and subharmonic solutions for second-order superlinear difference equations. Sci. China Math. 46(4), 506-515 (2003)

19. Guo, Z.M., Yu, J.S.: The existence of periodic and subharmonic solutions of subquadratic second order difference equations. J. Lond. Math. Soc. 68(2), 419-430 (2003) 
20. Gupta, C.P.: Existence and uniqueness theorems for the bending of an elastic beam equation. Appl. Anal. 26(4), 289-304 (1988)

21. Hale, J.K., Mawhin, J.: Coincidence degree and periodic solutions of neutral equations. J. Differ. Equ. 15(2), 295-307 (1974)

22. Han, G., Xu, Z.: Multiple solutions of some nonlinear fourth-order beam equations. Nonlinear Anal. 68(12), 3646-3656 (2008)

23. Henderson, J., Thompson, H.B.: Existence of multiple solutions for second-order discrete boundary value problems. Comput. Math. Appl. 43(10-11), 1239-1248 (2002)

24. Kocic, V.L., Ladas, G.: Global Behavior of Nonlinear Difference Equations of Higher Order with Applications. Kluwer Academic, Dordrecht (1993)

25. Li, F., Zhang, Q., Liang, Z.: Existence and multiplicity of solutions of a kind of fourth-order boundary value problem. Nonlinear Anal. 62(5), 803-816 (2005)

26. Liu, Y.J., Ge, W.G.: Twin positive solutions of boundary value problems for finite difference equations with $p$-Laplacian operator. J. Math. Anal. Appl. 278(2), 551-561 (2003)

27. Matsunaga, H., Hara, T., Sakata, S.: Global attractivity for a nonlinear difference equation with variable delay. Comput. Math. Appl. 41(5-6), 543-551 (2001)

28. Mawhin, J., Willem, M.: Critical Point Theory and Hamiltonian Systems. Springer, New York (1989)

29. Mickens, R.E.: Difference Equations: Theory and Application. Van Nostrand Reinhold, New York (1990)

30. Pankov, A., Zakhrchenko, N.: On some discrete variational problems. Acta Appl. Math. 65(1-3), 295-303 (2001)

31. Peterson, A., Ridenhour, J.: The $(2,2)$-disconjugacy of a fourth order difference equation. J. Differ. Equ. Appl. 1(1), 87-93 (1995)

32. Popenda, J., Schmeidel, E.: On the solutions of fourth order difference equations. Rocky Mt. J. Math. 25(4), 1485-1499 (1995)

33. Rabinowitz, P.H.: Minimax Methods in Critical Point Theory with Applications to Differential Equations. Amer. Math. Soc., Providence (1986)

34. Sharkovsky, A.N., Maistrenko, Y.L., Romanenko, E.Y.: Difference Equations and Their Applications. Kluwer Academic, Dordrecht (1993)

35. Shi, H.P., Ling, W.P., Long, Y.H., Zhang, H.Q.: Periodic and subharmonic solutions for second order nonlinear functional difference equations. Commun. Math. Anal. 5(2), 50-59 (2008)

36. Thandapani, E., Arockiasamy, I.M.: Fourth-order nonlinear oscillations of difference equations. Comput. Math. Appl. 42(3-5), 357-368 (2001)

37. Wang, H.Y.: On the existence of positive solutions for semilinear elliptic equations in the annulus. J. Differ. Equ. 109(1), 1-7 (1994)

38. Yan, J., Liu, B.: Oscillatory and asymptotic behavior of fourth order nonlinear difference equations. Acta Math. Sin. 13(1), 105-115 (1997)

39. Yao, Q.: Positive solutions of a nonlinear elastic beam equation rigidly fastened on the left and simply supported on the right. Nonlinear Anal. 69(5-6), 1570-1580 (2008)

40. Yu, J.S., Guo, Z.M.: Boundary value problems of discrete generalized Emden-Fowler equation. Sci. China Math. 49(10), 1303-1314 (2006)

41. Yu, J.S., Guo, Z.M.: On boundary value problems for a discrete generalized Emden-Fowler equation. J. Differ. Equ. 231(1), 18-31 (2006)

42. Zhou, Z., Yu, J.S., Chen, Y.M.: Periodic solutions of a $2 n$ th-order nonlinear difference equation. Sci. China Math. 53(1), 41-50 (2010)

43. Zou, W.M., Schechter, M.: Critical Point Theory and Its Applications. Springer, New York (2006) 